\title{
Composites Based Green Poly(L-Lactic Acid) and Dioctyl Phthalate: Preparation and Performance
}

\author{
Yan-Hua Cai, ${ }^{1,2}$ Li-Sha Zhao, ${ }^{1}$ and Yan-Hua Zhang ${ }^{1}$ \\ ${ }^{1}$ Chongqing Key Laboratory of Environmental Materials \& Remediation Technologies, Chongqing University of Arts and Sciences, \\ Chongqing 402160, China \\ ${ }^{2}$ School of Materials and Chemical Engineering, Chongqing University of Arts and Sciences, Yongchuan, Chongqing 402160, China
}

Correspondence should be addressed to Yan-Hua Cai; caiyh651@aliyun.com

Received 12 October 2014; Revised 30 January 2015; Accepted 6 February 2015

Academic Editor: P. J. Schubel

Copyright (C) 2015 Yan-Hua Cai et al. This is an open access article distributed under the Creative Commons Attribution License, which permits unrestricted use, distribution, and reproduction in any medium, provided the original work is properly cited.

\begin{abstract}
The effects of dioctyl phthalate (DOP) on performances of poly(L-lactic acid) (PLLA) were investigated in detail using optical depolarizer, X-ray diffraction, melt index instrument, and electronic tensile tester. Crystallization performance showed that the half time of overall PLLA crystallization $t_{1 / 2}$ decreased with increasing of crystallization temperature $\left(80^{\circ} \mathrm{C}\right.$ to $\left.105^{\circ} \mathrm{C}\right)$, but the $t_{1 / 2}$ of PLLA/DOP composites firstly decreased and then increased, and $t_{1 / 2}$ of PLLA/DOP exhibited minimum value at $85^{\circ} \mathrm{C}$. Compared to neat PLLA, 20\%DOP made the $t_{1 / 2}$ decrease from $7258.3 \mathrm{~s}$ to $265.4 \mathrm{~s}$. X-ray diffraction experiment further confirmed that DOP could accelerate the crystallization of PLLA. The fluidity of PLLA/DOP composites indicated that the melt mass flow rate firstly decreased and then greatly increased with increasing of DOP content. The mechanical performance showed that DOP could improve the general mechanical performance, and the elongation at break of PLLA/25\%DOP was about 30 times longer than that of neat PLLA.
\end{abstract}

\section{Introduction}

Dioctyl phthalate (DOP) as a conventional additive in the world has obtained broader attention due to its cheaper cost and more advanced plasticization ability. Thus, viscous DOP was widely used to improve the plasticization ability for polymer materials [1-3]. In addition, the presence of DOP also influences the crystallization and stability of the polymers $[4,5]$. Some literatures reported that the addition of DOP could decrease the degree of crystallization of the polymers, resulting from the increasing of macromolecule moving ability. For examples, Baltieri et al. [6] reported the influence of DOP on thermal properties of poly(3hydroxybutyrate). Experiment dates analysis indicated that DOP exhibited a good effect on the thermal properties of poly(3-hydroxybutyrate), and DOP made the glass transition temperature, melt point, and the degree of crystallinity decrease. Similarly, the results from Huang and his colleagues also confirmed that addition of DOP could make the degree of crystallization of PVC decrease [7].
Compared to other thermoplastic polymers, poly(L-lactic acid) (PLLA) produced from renewable sources has become the most important biomass thermoplastic polymers materials, because of its excellent lower energy consumption, biodegradation ability, processability, and biocompatibility $[8,9]$. Nowadays, PLLA and its composites have been applied to more and more fields to be as substitution of the traditional polymer such PP, PE, and PVC. However, it is necessary to further improve the performance of materials based PLLA to wide application., PLLA still has especially some disadvantages such as slow crystallization rate and poor dimensional stability to restrict the wider range application in daily life. Thus, many technologies and methods were used by researcher to improve the performance of PLLA. Fan et al. [10] reported that a novel organic compound $N, N^{\prime}$-bis(benzoyl)sebacic acid dihydrazide was synthesized to be as a nucleating agent for PLLA to improve the degree of crystallinity, the experiment results showed that $N, N^{\prime}$-bis(benzoyl)sebacic acid dihydrazide could enhance the crystallization rate and thermal 
stability of PLLA. 1.5\% $N, N^{\prime}$-bis(benzoyl)sebacic acid dihydrazide made the half time of overall PLLA crystallization decrease from $33.76 \mathrm{~min}$ to $2.36 \mathrm{~min}$ at $110^{\circ} \mathrm{C}$. Similarly, Wang and his colleagues [11] also synthesized a series of 1,3,5-trialkyl-benzenetricarboxylamides to use as nucleating agents of PLA, and compared to the neat PLLA, 1,3,5-trialkylbenzenetricarboxylamides made PLA exhibit a very short half time of over all crystallization in a wide region 5 from $90^{\circ} \mathrm{C}$ to $130^{\circ} \mathrm{C}$.

PLLA and DOP including ester group may improve the miscibility of PLLA and DOP. On the other hand, it is fact that DOP was used mainly for PVC plasticization $[1,12]$. Thus, the investigations are of very importance to investigate the effect of DOP on PLLA. In this paper, DOP was introduced into PLLA matrix to fabricate composites, and the crystallization behavior, fluidity, and mechanical performance were investigated in detail.

\section{Experiment}

2.1. Materials. PLLA (2002D) was purchased from Nature Works LLC (USA). DOP (analytical grade) was purchased from Beijing Chemical Reagents Company (Beijing, China).

2.2. Preparation of PLLA/DOP Composites. Firstly, PLLA was dried overnight at $35^{\circ} \mathrm{C}$ under vacuum, then the blending of PLLA and DOP was carried out using a counter-rotating mixer, and the similar operation process was reported in our previous work [13].

\subsection{Test}

2.3.1. X-Ray Diffraction (XRD). XRD of PLLA/DOP composites with different heating treatment was performed on a diffractometer using $\mathrm{Cu} K_{\alpha}$ radiation (wavelength, $1.54 \AA$ ) in the range of $2 \theta=10-60^{\circ}$ with scanning rate of $2^{\circ} / \mathrm{min}$.

2.3.2. Crystallization Measurement. Crystallization behavior of PLLA/DOP composites was investigated by optical depolarizer from $80^{\circ} \mathrm{C}$ to $105^{\circ} \mathrm{C}$.

2.3.3. Melt Index. The fluidity of melting PLLA/DOP composites was measured by melt index instrument, and the measurement temperature was $180^{\circ} \mathrm{C}$; load is $10 \mathrm{Kg}$.

2.3.4. Mechanical Performance Measurement. Mechanical performance measurement was performed on a M-4010 electronic tensile tester at the speed of $50 \mathrm{~mm} / \mathrm{min}$ at room temperature.

\section{Results and Discussion}

3.1. Isothermal Crystallization Behavior. Usually, the different crystallization behavior of PLLA with additive would directly affect the physical performance of PLLA, and the reason is that slow crystallization rate of the neat PLLA makes the molding process of materials based on PLLA become

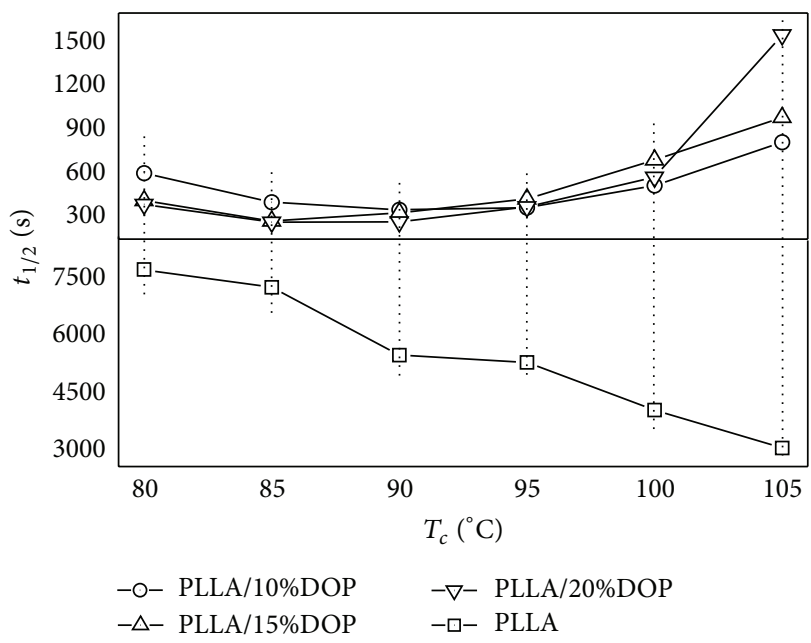

Figure 1: The $t_{1 / 2}$ of PLLA and PLLA/DOP with the crystallization temperature $T_{c}$.

difficult. On the other hand, the addition of the additive often can improve the crystallization properties of PLLA. In this study, we firstly investigate the effect of DOP on crystallization behavior of PLLA using optical depolarizer and XRD.

Figure 1 shows the effect of crystallization temperature and DOP content on crystallization of PLLA. As shown in Figure 1, the half time of overall PLLA crystallization $t_{1 / 2}$ is very long in the region from $80^{\circ} \mathrm{C}$ to $105^{\circ} \mathrm{C}$; the reason is that low crystallization temperature makes the movement ability of PLLA become difficulty, though low crystallization temperature easily makes PLLA aggregate to be nucleation, resulting in PLLA molecular chain not achieving regular arrangement. However, it is very clear that DOP significantly affects the crystallization of PLLA, and the addition of DOP can accelerate the crystallization of PLLA and makes the $t_{1 / 2}$ decrease. On the other hand, the $t_{1 / 2}$ of PLLA continuously decreases with increasing of crystallization temperature, which indicates that crystallization temperature is also one of the most important factors for crystallization of PLLA. However, the trend of PLLA/DOP composites is different from neat PLLA. With increasing of crystallization temperature, the $t_{1 / 2}$ of PLLA/DOP composites firstly decreases and then increases. And the $t_{1 / 2}$ has the minimum value at $85^{\circ} \mathrm{C}$. In addition, the $t_{1 / 2}$ of PLLA/DOP composites decreases in the region from $80^{\circ} \mathrm{C}$ to $90^{\circ} \mathrm{C}$ with increasing of DOP content. In contrast, the $t_{1 / 2}$ increases in the region from $95^{\circ} \mathrm{C}$ to $105^{\circ} \mathrm{C}$, indicating very complicated effect of DOP on crystallization of PLLA. Compared to neat PLLA, 20\%DOP makes the $t_{1 / 2}$ have the minimum value in $85^{\circ} \mathrm{C}$, and the $t_{1 / 2}$ decreases from $7258.3 \mathrm{~s}$ to $265.4 \mathrm{~s}$. That is to say, the $t_{1 / 2}$ for neat PLLA is about 27 times longer than that of PLLA with 20\%DOP.

$\mathrm{XRD}$ analysis of PLLA/DOP samples was carried out to further confirm the enhancement of DOP on crystallization of PLLA. Figure 2 shows the XRD curves of PLLA/DOP without heat treatment. It is observed that the diffraction peaks of PLLA and PLLA/DOP samples are very wide, which 


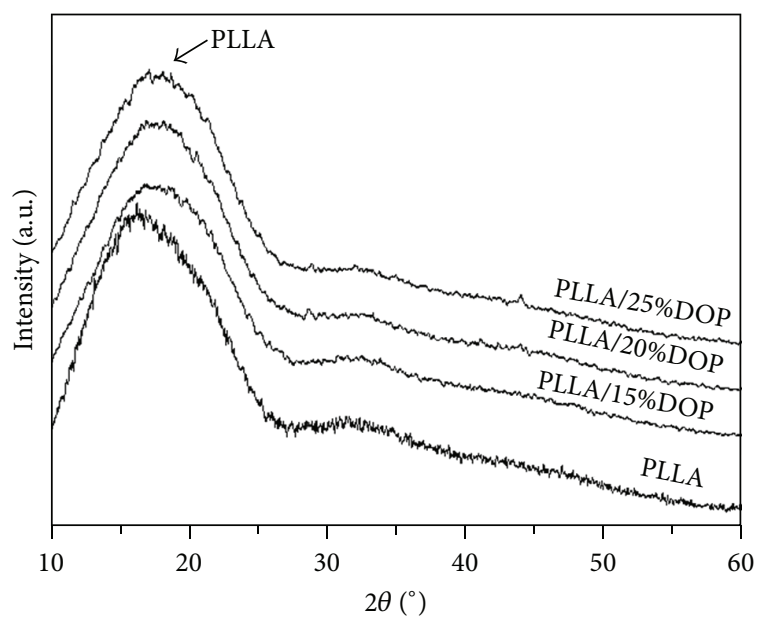

FIGURE 2: XRD curves of PLLA and PLLA/DOP samples.

indicates the most of PLLA matrix is noncrystal. Moreover, the diffraction peak of neat PLLA is sharper than that of PLLA/DOP; the possible reason is that the addition of DOP increases the plasticity of PLLA and makes PLLA form more noncrystal.

However, the XRD curve of PLLA/DOP samples after heat treatment is completely different from that of PLLA at room temperature. Figure 3 is the XRD curves of neat PLLA and PLLA/DOP samples after heat treatment. It is observed from Figure 3 that the diffraction peak of neat PLLA is still very wide after isothermal crystallization for 4 min at $85^{\circ} \mathrm{C}$, indicating the poor crystallization ability of neat PLLA. And the PLLA/DOP samples exhibit very strong diffraction peaks after the same heat treatment, resulting from forming more PLLA crystal. This result further confirms that DOP can accelerate the crystallization of PLLA; on the other hand, this result is consistent with the aforementioned isothermal crystallization results. As seen in Figure 3, the strongest diffraction peak of PLLA/DOP samples at $2 \theta=$ $16.6^{\circ}$ due to diffraction from (110) planes and other peaks at $2 \theta=14.7^{\circ}, 19.0^{\circ}$, and $22.2^{\circ}$ occurring from the (010) plane, (203) plane, and (205) plane, respectively [14]. In addition, the intensity of diffraction peak increases with increase of DOP content, but $25 \% \mathrm{DOP}$ makes the intensity of diffraction peak slightly decrease; this result also indicates effect of DOP on crystallization of PLLA is very complicated.

With increasing of crystallization time, as to the neat PLLA, the diffraction peak appears and the intensity of diffraction peak is very weak (see Figure 4 ). But the intensity of diffraction peak of PLLA/DOP samples is very strong, what is more, the intensity of diffraction peak of PLLA/DOP hardly change except for PLLA/15\%DOP, indicating the PLLA/20\%DOP and PLLA/25\%DOP exhibit the maximum degree of crystallization after heat treatment for $8 \mathrm{~min}$ at $85^{\circ} \mathrm{C}$. And the intensity of diffraction peak of PLLA $/ 15 \% \mathrm{DOP}$ also achieves the maximum after further increasing crystallization time (see Figure 5). However, the intensity of diffraction peak of neat PLLA still increases, resulting from

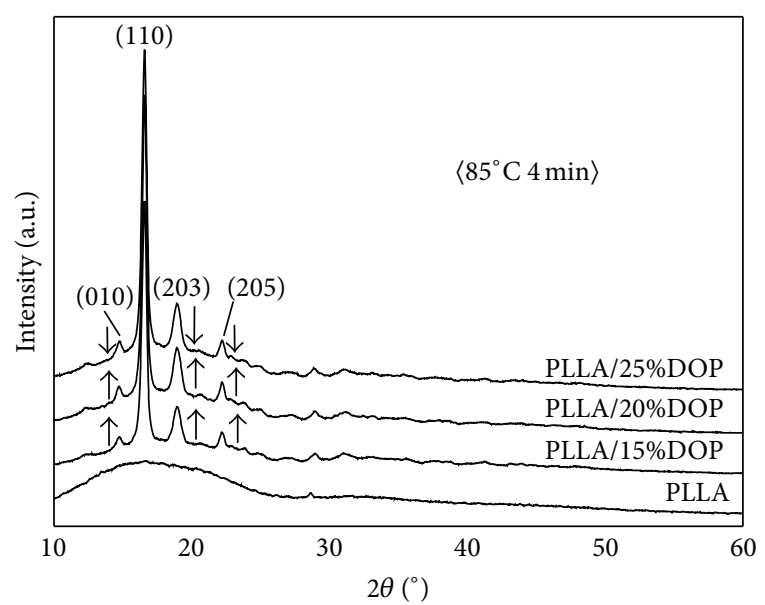

FIgURE 3: XRD curves of PLLA and PLLA/DOP samples for $4 \mathrm{~min}$ at $85^{\circ} \mathrm{C}$.

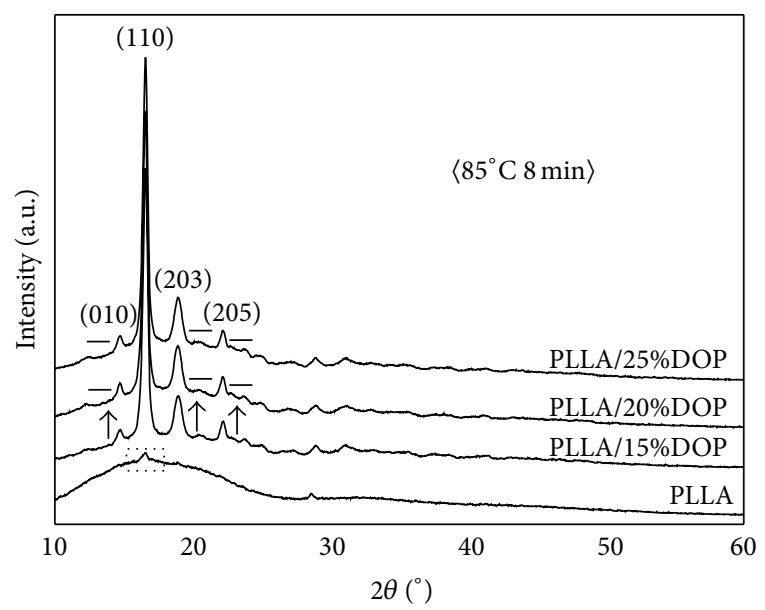

FIgURE 4: XRD curves of PLLA and PLLA/DOP samples for 8 min at $85^{\circ} \mathrm{C}$.

increasing crystal of neat PLLA, which also further confirms that the crystallization ability of neat PLLA is very poor.

3.2. Fluidity and Mechanical Performance. As mentioned above, the addition of DOP would change the movement ability of PLLA molecular chain. Thus, there is no doubt that the addition of DOP also would affect the fluidity of PLLA. Figure 6 shows the effect of DOP on the melt mass flow rate (MFR) of PLLA. With increasing of DOP content, the MFR firstly decreases and then greatly increases. The reason may be that a small amount of DOP only slightly improves the movement ability of PLLA molecular chain, resulting in twine of macromolecule segment and affecting the fluidity of PLLA. However, upon a large load DOP, the movement ability and the plasticization ability of macromolecule segment significantly increase and the MFR also increases during process of PLLA. Overall, the addition of DOP can effectively improve the fluidity of PLLA.

Mechanical performance is the most important index to measure plasticization ability of additive. Table 1 shows the 


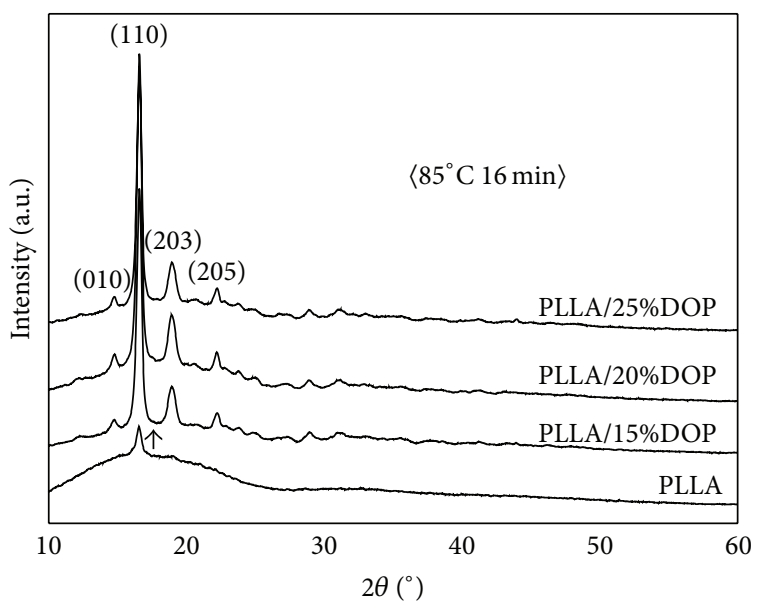

Figure 5: XRD curves of PLLA and PLLA/DOP samples for $16 \mathrm{~min}$ at $85^{\circ} \mathrm{C}$.

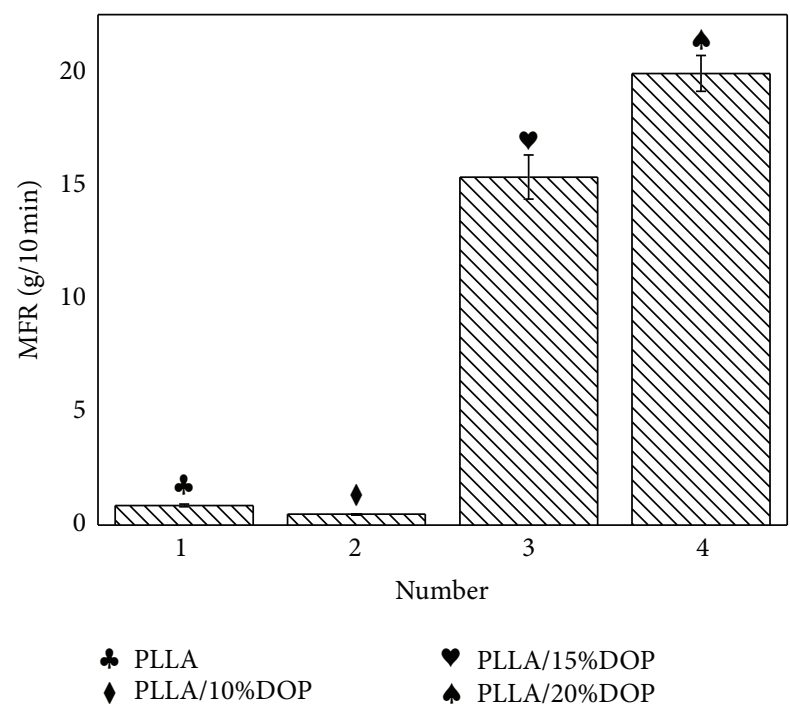

Figure 6: The effect of DOP content on MFR of PLLA.

influence of DOP on the mechanical performance of PLLA. With increasing of DOP content, the tensile strength of PLLA decreases, and the elongation at break of PLLA increases; the results are similar to the effect of DOP on mechanical properties of other thermoplastic polymers [3]. It is obvious from Table 1 that the tensile strength of PLLA is about 2 times than that of PLLA/25\%DOP, and the elongation at break of PLLA/25\%DOP is about 30 times than that of PLLA. In a word, to some extent plasticizer DOP can improve the general mechanical performance.

\section{Conclusion}

PLLA/DOP composites were fabricatedby melt blending technology, and the physical performances of PLLA/DOP composites were investigated. The crystallization behavior indicated that the introduction of DOP could significantly increase the crystallization rate of PLLA through optical
TABLE 1: Mechanical properties of PLLA and PLLA/DOP samples.

\begin{tabular}{lcc}
\hline Sample & Tensile strength $(\mathrm{MPa})$ & Elongation at break $(\%)$ \\
\hline PLLA & $47.6 \pm 2.4$ & $5.2 \pm 0.63$ \\
PLLA/10\%DOP & $40.6 \pm 1.1$ & $6.98 \pm 0.56$ \\
PLLA/15\%DOP & $35 \pm 1.9$ & $12.18 \pm 0.81$ \\
PLLA/20\%DOP & $27.4 \pm 0.9$ & $38.99 \pm 0.85$ \\
PLLA/25\%DOP & $20.8 \pm 1.5$ & $155.98 \pm 1.75$ \\
\hline
\end{tabular}

depolarizer and XRD. The melt index and mechanical experiment showed DOP can effectively improve the fluidity and the general mechanical performance of PLLA. In a word, the PLLA/DOP composites may be a promising application polymer material.

\section{Conflict of Interests}

The authors declare that there is no conflict of interests regarding the publication of this paper.

\section{Acknowledgments}

This work was supported by National Natural Science Foundation of China (Project no. 51403027), China Postdoctoral Science Foundation (Project no. 2013M531937), Natural Science Foundation Project of CQ CSTC (Project no. cstc2012jjA50001), Foundation of Chongqing Municipal Education Commission (Project no. KJ131202), and Chongqing University of Arts and Sciences (Project no. R2013CH11).

\section{References}

[1] G. Szarka and B. Iván, “Thermal properties, degradation and stability of poly(vinyl chloride) predegraded thermooxidatively in the presence of dioctyl phthalate plasticizer," Journal of Macromolecular Science, Part A: Pure and Applied Chemistry, vol. 50, no. 2, pp. 208-214, 2013.

[2] Y.-T. Shieh and C.-M. Liu, "Influences of contents and molecular weights of LDPE on DOP plasticization of PVC," Journal of Applied Polymer Science, vol. 83, no. 12, pp. 2548-2555, 2002.

[3] M.-S. Zhou, Z.-J. Sun, D.-J. Yang, J.-H. Huang, and X.-Q. Qiu, "The effect of plasticizer on the properties of alkali lignin/HDPE composites," Acta Polymerica Sinica, vol. 2, pp. 210-217, 2014.

[4] G. Szarka and B. Iván, "Degradative transformation of poly(vinyl chloride) under mild oxidative conditions," ACS Symposium Series, vol. 1004, pp. 219-226, 2009.

[5] S.-L. Yang, Z.-H. Wu, B. Meng, and W. Yang, "The effects of dioctyl phthalate plasticization on the morphology and thermal, mechanical, and rheological properties of chemical crosslinked polylactide," Journal of Polymer Science, Part B: Polymer Physics, vol. 47, no. 12, pp. 1136-1145, 2009.

[6] R. C. Baltieri, L. H. I. Mei, and J. Bartoli, "Study of the influence of plasticizers on the thermal and mechanical properties of poly(3-hydroxybutyrate) compounds," Macromolecular Symposia, vol. 197, pp. 33-44, 2003.

[7] Z.-M. Huang, Y.-Z. Bao, Z.-X. Weng, and Z.-R. Pan, "Study on crystallization behavior of poly(vinyl chloride)," Polymer Materials Science and Engineering, vol. 14, no. 4, pp. 78-81, 1998. 
[8] Y.-H. Cai, "Synthesis and nucleating effect of $N, N^{\prime}$-bis(benzoyl) terephthalic acid dihydrazide," Journal of the Chemical Society of Pakistan, vol. 34, no. 3, pp. 709-712, 2012.

[9] J. Tuominen, J. Kylma, A. Kapanen, O. Venelampi, M. Itävaara, and J. Seppälä, "Biodegradation of lactic acid based polymers under controlled composting conditions and evaluation of the ecotoxicological impact," Biomacromolecules, vol. 3, no. 3, pp. 445-455, 2002.

[10] Y. Fan, Z. Yu, Y. Cai et al., "Crystallization behavior and crystallite morphology control of poly(L-lactic acid) through $N, N^{\prime}$ bis(benzoyl)sebacic acid dihydrazide," Polymer International, vol. 62, no. 4, pp. 647-657, 2013.

[11] T. Wang, Y. Yang, C. Zhang, Z. Tang, H. Na, and J. Zhu, "Effect of 1,3,5-trialkyl-benzenetricarboxylamide on the crystallization of poly(lactic acid)," Journal of Applied Polymer Science, vol. 130, no. 2, pp. 1328-1336, 2013.

[12] A. E. A. Toubal, N. Fertikh, S. Ali-Mokhnache, and D. Messadi, "Evaluation of a mathematical model of dioctyl phthalate plasticizer migration from PVC discs into colza oil," Iranian Polymer Journal, vol. 14, no. 8, pp. 721-728, 2005.

[13] Y.-H. Cai, "Nucleation, melting behaviour and mechanical properties of poly(L-lactic acid) affected by the addition of $N, N^{\prime}$-bis(benzoyl) suberic acid dihydrazide," South African Journal of Chemistry, vol. 64, pp. 115-119, 2011.

[14] W.-D. Hergeth, W. Lebek, E. Stettin, K. Witkowski, and K. Schmutzler, "Particle formation in emulsion polymerization, 2. Aggregation of primary particles," Die Makromolekulare Chemie, vol. 193, no. 7, pp. 1607-1621, 1992. 

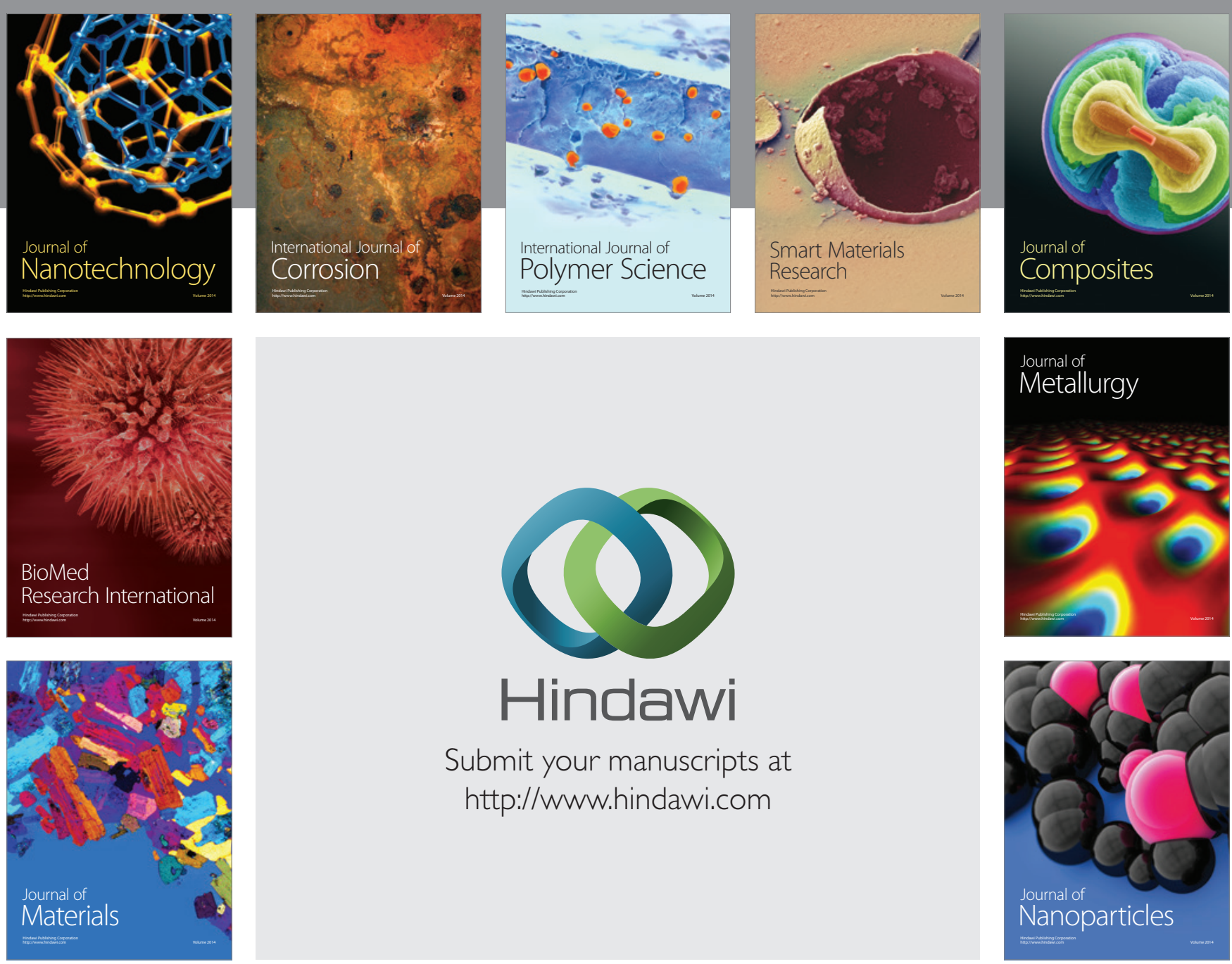

Submit your manuscripts at http://www.hindawi.com
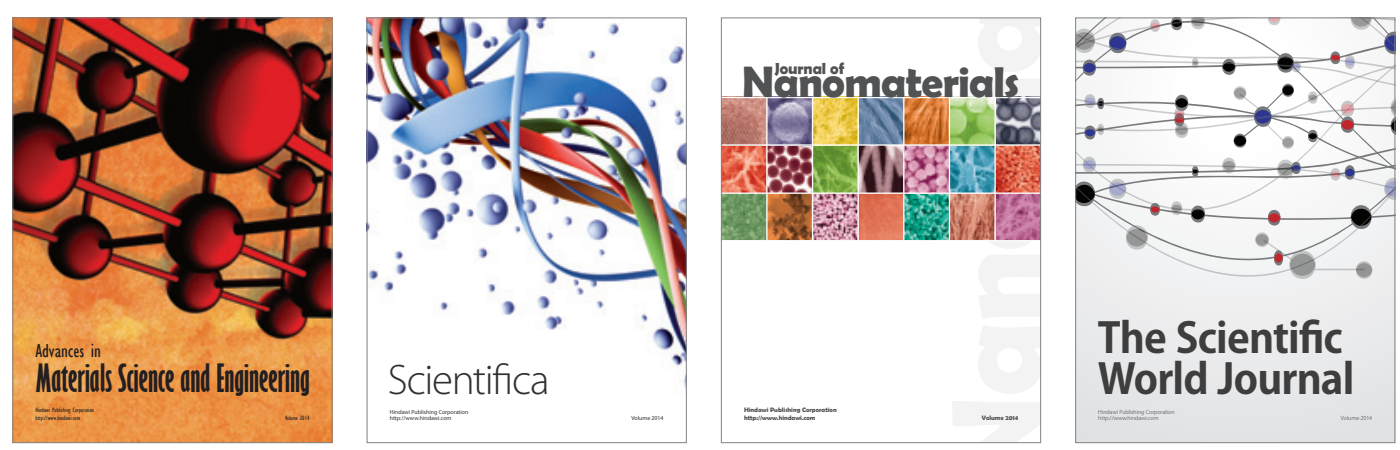

\section{The Scientific World Journal}
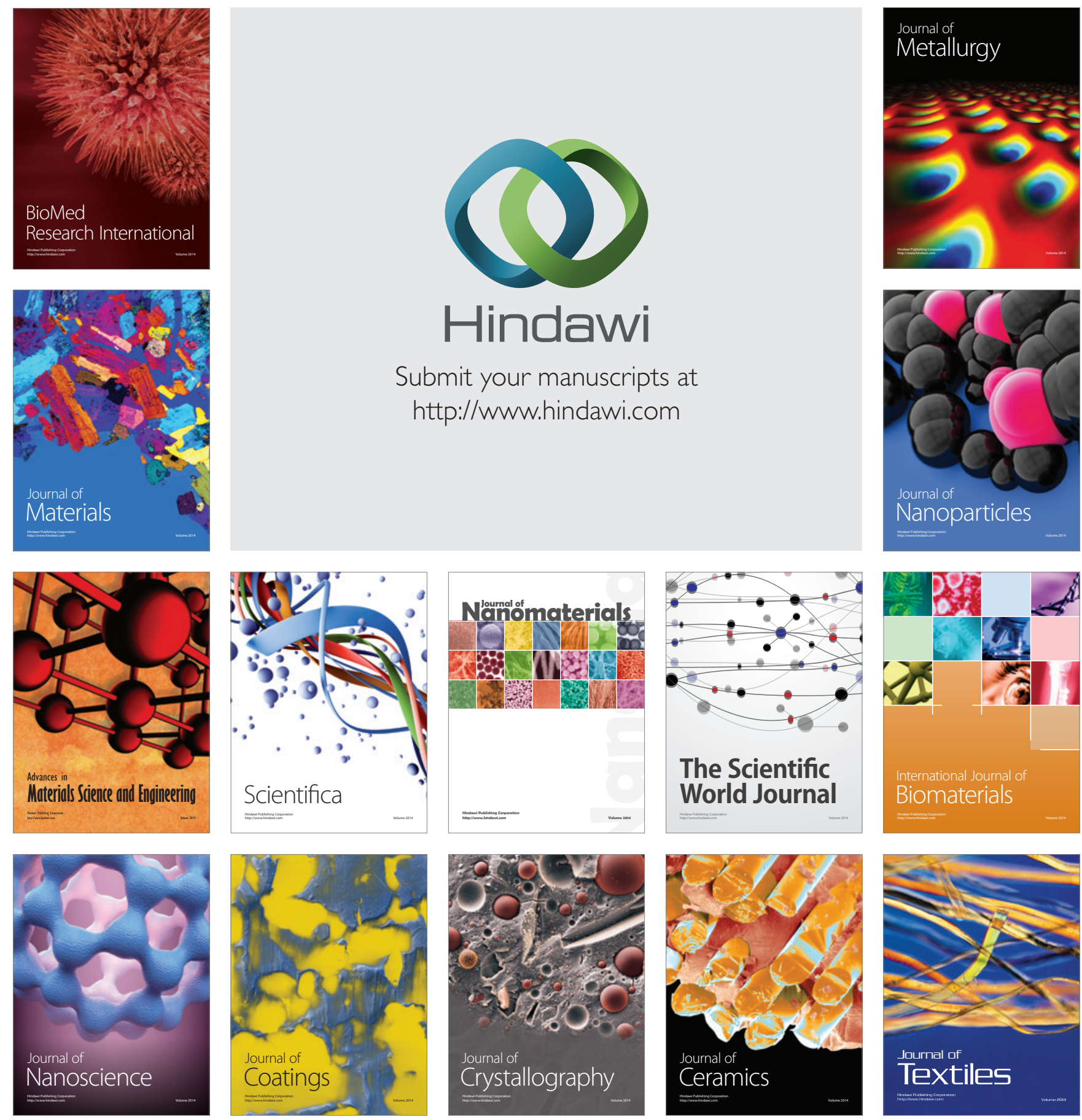